Meta

Journal des tradlucteurs

Translators' Journal

\title{
Équivalence lexicale et traduction
}

\section{Christine Bagge}

Volume 35, numéro 1, mars 1990

Actes du colloque international « La traduction proligère "

URI : https://id.erudit.org/iderudit/001942ar

DOI : https://doi.org/10.7202/001942ar

Aller au sommaire du numéro

Éditeur(s)

Les Presses de l'Université de Montréal

ISSN

0026-0452 (imprimé)

1492-1421 (numérique)

Découvrir la revue

Citer cet article

Bagge, C. (1990). Équivalence lexicale et traduction. Meta, 35(1), 61-67.

https://doi.org/10.7202/001942ar d'utilisation que vous pouvez consulter en ligne.

https://apropos.erudit.org/fr/usagers/politique-dutilisation/ 


\section{ÉQUIVALENCE LEXICALE ET TRADUCTION}

CHRISTINE BAGGE

Université Laurentienne, Sudbury, Canada

\section{INTRODUCTION}

La lexicographie est une activité pratique qui remonte à au moins trois cents ans (Hartmann 1983:5), mais, dans le monde occidental, ce n'est qu'au début du XVI $\mathrm{X}^{\mathfrak{e}}$ siècle que l'on entreprit pour la première fois de décrire le vocabulaire de façon systématique. Or, d'après Cohen (1962: 498-499), cet événement est le résultat de réalités culturelles et s'est effectué tout d'abord dans une optique comparative. À cette époque, le latin avait perdu son statut de première langue, mais il demeurait la langue des intellectuels. Les glossaires bilingues latin-français et français-latin et les dictionnaires polyglottes firent alors leur apparition. Comme le soulignent Anderson (1972:1) et Wiezell (1975: 132), donc, et contrairement à ce que l'on aurait tendance à penser a priori, historiquement le dictionnaire bilingue a précédé le monolingue.

À ce sujet, il est intéressant de noter deux réalités. D'une part, le dictionnaire bilingue (siège d'équivalences lexicales) n'est pas le résultat pratique d'une théorie longuement élaborée mais est né spontanément pour répondre à un besoin précis. D'autre part, ce besoin a été créé par la nécessité de traduire. Or, bien que la théorie de la traduction soit passée par divers stades au cours de son histoire (par exemple l'éternelle question de la fidélité), rien ne laisse supposer que l'acte de traduire ait été fondamentalement différent au XVI ${ }^{\mathrm{e}}$ siècle de ce qu'il est encore aujourd'hui. De plus, les problèmes que soulève l'équivalence lexicale sont au cœur de toutes les discussions théoriques, quelle que soit l'optique adoptée (comparative aussi bien que textologique), que la traduction soit considérée comme un résultat (voir Vinay et Darbelnet) ou comme une activité (voir Delisle). Toutes ces observations constituent donc un indice assez probant que l'équivalence lexicale joue un rôle important dans l'activité traduisante. La question qui se pose est de savoir comment réconcilier cette constatation avec le fait sur lequel, depuis longtemps et aujourd'hui plus que jamais ( $c f$. la textologie), la théorie met l'accent: la traduction ne consiste pas simplement à remplacer des mots par des mots.

\section{DÉFINITION DE LA NOTION D'ÉQUTVALENCE LEXICALE}

Dans le domaine linguistique, la notion d'équivalence désigne une similarité de sens. En traduction, cette relation s'établit entre deux langues et, lorsqu'il s'agit d'équivalence lexicale, elle concerne deux mots ou deux expressions. Avant d'essayer de décider quel rôle l'équivalence lexicale joue dans le processus traductionnel, il est donc nécessaire de préciser ce que l'on entend dans ce contexte par mots ou expressions (unité minimale) et sens.

\subsection{UNITÉ MINIMALE}

La lexicographie est avant tout un domaine pratique qui, jusqu'à récemment, théorisait peu sur sa méthode (Weinreich 1975: 26); c'est pourquoi la définition de l'unité minimale qu'elle étudie, le lexème, n'était pas jusqu'à présent très précise. La pratique lexicographique habituelle consiste à utiliser des catégories syntaxiques (noms, verbes, 
adjectifs, etc.) pour justifier la nécessité d'une entrée distincte et à grouper tous les sens reliés entre eux par le processus de la métaphore. Récemment, cependant, Cruse (1986: 76-80) a tenté de circonscrire plus exactement la nature du lexème. D'après lui, le lexème est «a family of lexical units; a lexical unit is the union of a single sense with a lexical form; a lexical form is an abstraction from a set of word forms (or alternatively - it is a family of word forms) which differ only in respect of inflections» (1986: 80). La figure 1 représente schématiquement cette définition et la figure 2 l'illustre par un exemple. La clarification de la distinction qui existe entre ces trois notions - lexème, unité lexicale et forme lexicale - facilite beaucoup la discussion. Elle n'aide pas à décider à quel lexème appartient une certaine unité lexicale, mais il s'agit là d'un problème intralinguistique qui n'intervient pas au niveau de l'équivalence, où on ne compare qu'un seul sens à la fois. Si l'on adopte la terminologie de Cruse, l'unité lexicale semble donc être l'unité sémantique opérationnelle la plus adaptée aux besoins de l'équivalence lexicale. Elle est en effet constituée d'au moins un mot - pris dans son sens d' «unité de texte inscrite entre deux blancs graphiques» (Larose 1987:25) - et n'a qu'un sens. Ce qu'il reste à déterminer, c'est dans quelles circonstances un syntagme, pris dans l'acception restreinte de groupe de mots qui se suivent avec un sens, forme une unité lexicale.

Le statut linguistique du mot a fait couler beaucoup d'encre dans le domaine de la linguistique. En traduction, par contre, ce statut va de soi, puisque le mot est écrit et se définit donc comme ci-dessus: unité de texte entre deux blancs. La difficulté vient alors du fait qu'un signifié, au sens que lui a donné Saussure, nécessite souvent un signifiant composé de plus d'un mot. Ceci n'est pas surprenant, la décision d'avoir un ou plusieurs mots semblant être souvent totalement arbitraire. En français, par exemple, Vinay et Darbelnet (1977: 36) mentionnent la coexistence de «bon sens», «non-sens» et «contresens». C'est donc sur la question de savoir ce qui constitue une unité lexicale dans une analyse interlinguistique (souvent nommée «unité de traduction» et pour laquelle Larose (1987: 218) a relevé neuf appellations différentes) que la théorie de la traduction a été prolifique. En fait, la définition qu'en donnent Vinay et Darbelnet (1977: 16) suffira dans la mesure où les discussions qui ont suivi sur ce sujet (voir Tatilon 1982) ont modifié leur taxinomie ou en ont proposé une autre mais sans remettre en question la définition de base: «le plus petit segment de l'énoncé dont la cohésion des signes est telle qu'ils ne doivent pas être traduits séparément». Théoriquement, donc, ces unités peuvent aller du simple mot à toute une phrase dans le cas des expressions idiomatiques. Ce fait est très nettement visible dans tous les dictionnaires bilingues.

L'unité minimale qui permet d'établir une équivalence lexicale entre deux langues est donc une unité lexicale (telle que définie par Cruse) qui :

1) est formée d'au moins un mot;

2) n'a qu'un sens, et

3) si elle contient plus d'un mot, forme un ensemble sémantique cohérent indivisible.

\subsection{SENS D'UNE UNITÉ LEXICALE}

La théorie de la traduction laisse aux philosophes du langage (par exemple Wiggins 1971 et Alston 1971) le soin de décider si les mots ont un sens par eux-mêmes. Elle se contente d'étudier la nature du sens des mots tels qu'ils se trouvent dans leur contexte puisque la traduction a toujours pour objet des textes. D'autre part, elle fait la distinction entre ce qu'elle nomme la signification linguistique d'un mot et son sens contextuel. Pour justifier cette opposition, elle invoque la dichotomie saussurienne entre la langue et la parole. Comme l'explique par exemple Delisle (1984: 58): «la signification [linguistique] d'un mot est ce à quoi il renvoie dans le système abstrait de la langue. Tout mot 
peut avoir plus d'une signification. Ces diverses «acceptions» sont perceptibles hors contexte, c'est-à-dire indépendamment de toute énonciation concrète, et c'est pourquoi il est possible de les compiler dans les ouvrages lexicographiques». Le sens contextuel d'un mot, par contre, «est ce à quoi un signe renvoie lorsqu'il s'insère dans un énoncé concret, dans une séquence linguistique issue d'un acte individuel de parole». Autrement dit, pour chaque unité lexicale, le traducteur aurait à sa disposition une équivalence de signification qu'il pourrait en général trouver dans le dictionnaire bilingue et une équivalence de sens qu'il devrait créer lui-même dans les cas où la signification linguistique et le sens contextuel ne concordent pas.

Mettre l'accent de cette façon sur la différence qui existe entre l'équivalence de signification (consignée dans le dictionnaire) et l'équivalence de sens (la façon dont l'unité lexicale est traduite dans le contexte) est fort utile sur le plan pédagogique et pratique, mais n'est pas justifiable au niveau de la théorie de la traduction. Il est en effet maintenant bien établi par les philosophes du langage, même lorsque leurs vues divergent sur d'autres points ( $c f$. Wiggins $1971: 16$, et Alston 1971:36), que le sens d'un mot est la contribution constante qu'il fait au sens de toute phrase dans laquelle il apparaît avec ce sens. Cette théorie trouve ses fondements dans le fait que, dans une langue donnée, l'ensemble des mots est fini et l'ensemble des phrases infini et que pourtant tout locuteur peut comprendre des phrases qu'il n'a jamais vues ou entendues auparavant. Comme l'expliquent Katz et Fodor (1963: 171-172), il doit donc exister une méthode systématique pour construire des phrases à partir d'éléments de base. Le sens des mots fait partie de ces éléments. Sans entrer plus en détail dans la discussion de cette question d'ordre philosophique, il est possible d'adapter cette définition au contexte de l'équivalence lexicale et de dire que, puisque l'unité lexicale n'a qu'un sens (cf. 1.1.), le sens d'une unité lexicale est la contribution constante qu'elle fait au sens de toute phrase dans laquelle elle apparaît. Dans ces conditions, donc, la signification linguistique est tout aussi contextuelle que le sens. Elle est le sens le plus usuel du lexème et appartient à une unité lexicale distincte; elle n'est pas hors contexte, elle correspond au contexte le plus usuel. Comme l'exprime Saussure (1973: 30), la langue est «un trésor déposé par la pratique de la parole dans les sujets appartenant à une même communauté». Lorsqu'un lexicographe établit la signification linguistique d'un lexème, sa première tâche consiste à le replacer dans autant de contextes que possible et à établir un certain nombre d'unités lexicales. Pour des raisons pratiques - par exemple le manque de place - il lui est nécessaire de privilégier le sens le plus usuel aux dépens des autres. Cependant, ces contraintes pratiques ne donnent pas à ce sens un statut particulier sur le plan théorique dans le cadre de la traduction. Au niveau de la langue, les unités lexicales n'ont que des virtualités de signification, pour reprendre l'expression de M. Lederer (1973: 8), et la seule façon de représenter la signification linguistique d'un lexème serait d'avoir une liste exhaustive de toutes les significations possibles de ce lexème. Bien que cette liste ne soit pas infinie (contrairement à ce que dit par exemple Delisle (1984: 134)) puisque la communication est possible, elle est pratiquement irréalisable. Il n'en demeure pas moins que théoriquement le sens d'une unité lexicale est toujours et linguistique, et contextuel. D'une part, tous les sens possibles d'un lexème existent virtuellement au niveau de la langue, sinon il serait impossible que plusieurs locuteurs comprennent la même phrase. D'autre part, tous ces sens sont spécifiques au contexte dans lequel ils apparaissent.

\section{PLACE DE L'ÉQUiVALENCE LEXICALE AU SEIN de L'ACTIVITÉ TRADUISANTE}

Définie telle qu'elle vient de l'être (similarité de sens de deux unités lexicales appartenant à deux langues différentes), l'équivalence lexicale est au centre de l'activité traduisante, comme le montre la figure 3 . Elle fait partie du bagage cognitif dont dispose 
le traducteur. Il est à noter que ce tableau impose un certain ordre dans les trois étapes du processus mais que, comme le fait remarquer Delisle (1984: 78), «la réflexion avance par étapes successives, mais sans nécessairement suivre une trajectoire rectiligne».

\subsection{PHRASE ÉCRITE DANS LA LANGUE DE DÉPART}

Lorsqu'il s'agit de traduire un texte, l'opération traduisante s'effectue en général une phrase à la fois sans que l'on perde le texte de vue. Dans la pratique, il est plutôt question de traduction "phrase à phrase» dont l'objectif est de parvenir, de proche en proche, à une traduction «texte à texte» (Larose 1987: 23).

\subsection{COMPRÉHENSION}

Les théoriciens et les praticiens s'accordent pour dire que la première étape de la tâche du traducteur consiste à comprendre l'énoncé à traduire. Comme l'explique Moskowitz (1973: 74), pour assurer l'invariance de l'information «le traducteur doit comprendre le message dans la langue source, faute de quoi il ne pourra pas émettre un message équivalent et compréhensible dans la langue cible». Étant donné que ce processus se passe dans le cerveau, il est toujours présomptueux de vouloir l'expliquer. Il est néanmoins possible d'énumérer les données qui interviennent dans cette opération:

1) le contexte (le traducteur travaillant toujours sur un texte écrit, le contexte sera avant tout linguistique);

2) les structures grammaticales et syntaxiques de la phrase;

3) le style;

4) l'apport de la connaissance du monde extra-linguistique;

5) les virtualités de sens des lexèmes.

\subsection{ANALYSE TRADUISANTE}

De la même façon que, comme l'a mentionné Wiggins (1971: 18), aucun locuteur n'apprend à produire ou à comprendre le nombre infini de phrases qu'il peut produire ou comprendre en apprenant leurs sens un par un, aucun traducteur n'apprend à traduire le nombre infini de phrases qu'il peut traduire en apprenant leurs traductions une par une. Étant donné qu'il y a un nombre infini de phrases anglaises, par exemple, à traduire, la traduction de ces phrases ne peut se faire qu'à l'aide d'une méthode de décomposition systématique en unités minimales de traduction - unités lexicales - et, sauf dans les cas rares où la phrase est traduite «en bloc» (par exemple les expressions idiomatiques) et devient alors elle-même une unité lexicale, chaque unité lexicale sera traduite séparément. Il ne s'agira pas pour le traducteur de faire du mot à mot à l'aide d'un dictionnaire, mais de cerner de près le sens de chaque unité lexicale dans le contexte en question et de lui trouver un équivalent, non pas formel, mais sémantique, dans la langue cible. Autrement dit, l'activité traduisante n'est possible que parce qu'il existe dans la langue cible des équivalents aux unités lexicales de la langue source.

\subsection{ACTION}

La dernière étape du processus traductionnel consiste à reformuler la phrase de départ en une phrase en langue d'arrivée. Pour effectuer cette tâche, le traducteur utilise les équivalences lexicales qu'il a établies et tient compte de la structure grammaticale de la langue d'arrivée et du style choisi en fonction de celui du texte en langue source.

\section{CONCLUSION}

Souligner l'importance du rôle de l'équivalence lexicale dans le processus traductionnel ne signifie pas épouser la notion de langue-nomenclature qui impliquerait 
que chaque mot a un équivalent dans l'autre langue, puisque la délimitation d'une unité lexicale n'est pas dictée par des contraintes formelles mais par des critères sémantiques. Ce n'est pas non plus réduire la traduction à un simple transcodage. De la même façon que jusqu'à récemment la théorie de la traduction avait pour objet le résultat plutôt que le processus, l'équivalence lexicale était en général considérée comme une équation figée consignée dans le dictionnaire bilingue. Mais au sein de l'activité traduisante, il s'agit en fait d'une activité exégétique telle que décrite pan exemple par Delisle (1984: 101-105). Par contre, donner à l'équivalence une place prépondérante permet peut-être de trouver un juste milieu entre les deux pôles traditionnels que sont le simple transcodage et la traduction libre. Dans cette optique, en effet, la tâche du traducteur ne consiste ni à faire du mot à mot ni à laisser totalement de côté la forme de la langue source. Le traducteur doit (1) délimiter les unités lexicales; (2) déterminer oe que chacune d'entre elles apporte au sens de la phrase; (3) exprimer cette contribution en un équivalent dans la langue cible. Ceci n'implique pas nécessairement que les équivalents aient la même forme ou emploient le même procédé ; il n'y a pas forcément, par exemple, concordance entre grammaire et moyens lexicaux. Comme le dit Delisle (1984: 68): «La justesse d'une équivalence se mesure à l'adéquation des concepts à raccorder, non à la similitude ou à la dissemblance des formes qui les expriment.» Ceci ne sous-entend pas non plus qu'il n'y a qu'une solution possible à un problème donné, puisque le fait que la synonymie existe dans la langue cible n'est pas remis en question.

Deux facteurs semblent donc obscurcir le rôle de l'équivalence lexicale: (1) la forme et (2) le sens le plus usuel. L'unité lexicale n'a pas toujours les mêmes limites qu'un mot orthographique et n'a pas automatiquement une forme similaire dans une autre langue; de plus les lexèmes sont beaucoup plus polysémiques que ne peut le reconnaître le dictionnaire. Il n'en demeure pas moins que l'équivalence lexicale, en tant qu'activité, se trouve au cœur du processus traductionnel.

Figure 1 : lexème / unité lexicale / forme lexicale

\begin{tabular}{|c|c|c|c|}
\hline \multirow{2}{*}{$\begin{array}{l}\text { unité } \\
\text { lexicale } 1 \\
\text { unité } \\
\text { lexicale } 2\end{array}$} & $\begin{array}{l}\text { sens } 1 \\
\text { (forme du mot } 1 \text { ) }\end{array}$ & $\begin{array}{l}\text { sens } 1 \\
\text { (forme du mot 2) }\end{array}$ & $\begin{array}{l}\text { sens } 1 \\
\text { (forme du mot } n \text { ) }\end{array}$ \\
\hline & $\begin{array}{l}\text { sens } 2 \\
(\text { forme du } \operatorname{mot} 1)\end{array}$ & $\begin{array}{l}\text { sens } 2 \\
\text { (forme du mot } 2 \text { ) }\end{array}$ & $\begin{array}{l}\text { sens } 2 \\
\text { (forme du mot } n \text { ) }\end{array}$ \\
\hline \multirow[b]{2}{*}{$\begin{array}{l}\text { unité } \\
\text { lexicale } m\end{array}$} & $\mathfrak{x}^{\prime}$ & 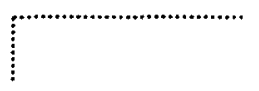 & 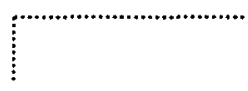 \\
\hline & $\begin{array}{l}\text { sens } m \\
\text { (forme du mot } 1 \text { ) }\end{array}$ & $\begin{array}{l}\text { sens } m \\
\text { (forme du mot } 2 \text { ) }\end{array}$ & $\begin{array}{l}\text { sens } m \\
(\text { forme du mot } n \text { ) }\end{array}$ \\
\hline
\end{tabular}


Figure 2 : lexème / unité lexicale / forme lexicale Exemple : lexème $=$ table

table

\begin{tabular}{|c|c|c|}
\hline $\begin{array}{l}\text { unité } \\
\text { lexicale } 1\end{array}$ & $\begin{array}{c}\text { meuble } \\
\text { (table) }\end{array}$ & $\begin{array}{c}\text { meuble } \\
\text { (tables) }\end{array}$ \\
\hline $\begin{array}{l}\text { unité } \\
\text { lexicale } 2\end{array}$ & $\begin{array}{l}\text { nourriture } \\
\text { (table) }\end{array}$ & $\begin{array}{l}\text { nourriture } \\
\text { (tables) }\end{array}$ \\
\hline & : & לְ. \\
\hline $\begin{array}{l}\text { unité } \\
\text { lexicale } m\end{array}$ & $\begin{array}{l}\text { tableau } \\
\text { (table) }\end{array}$ & $\begin{array}{l}\text { tableau } \\
\text { (tables) }\end{array}$ \\
\hline
\end{tabular}

Figure 3 : Place de l'équivalence lexicale au sein de l'activité traduisante

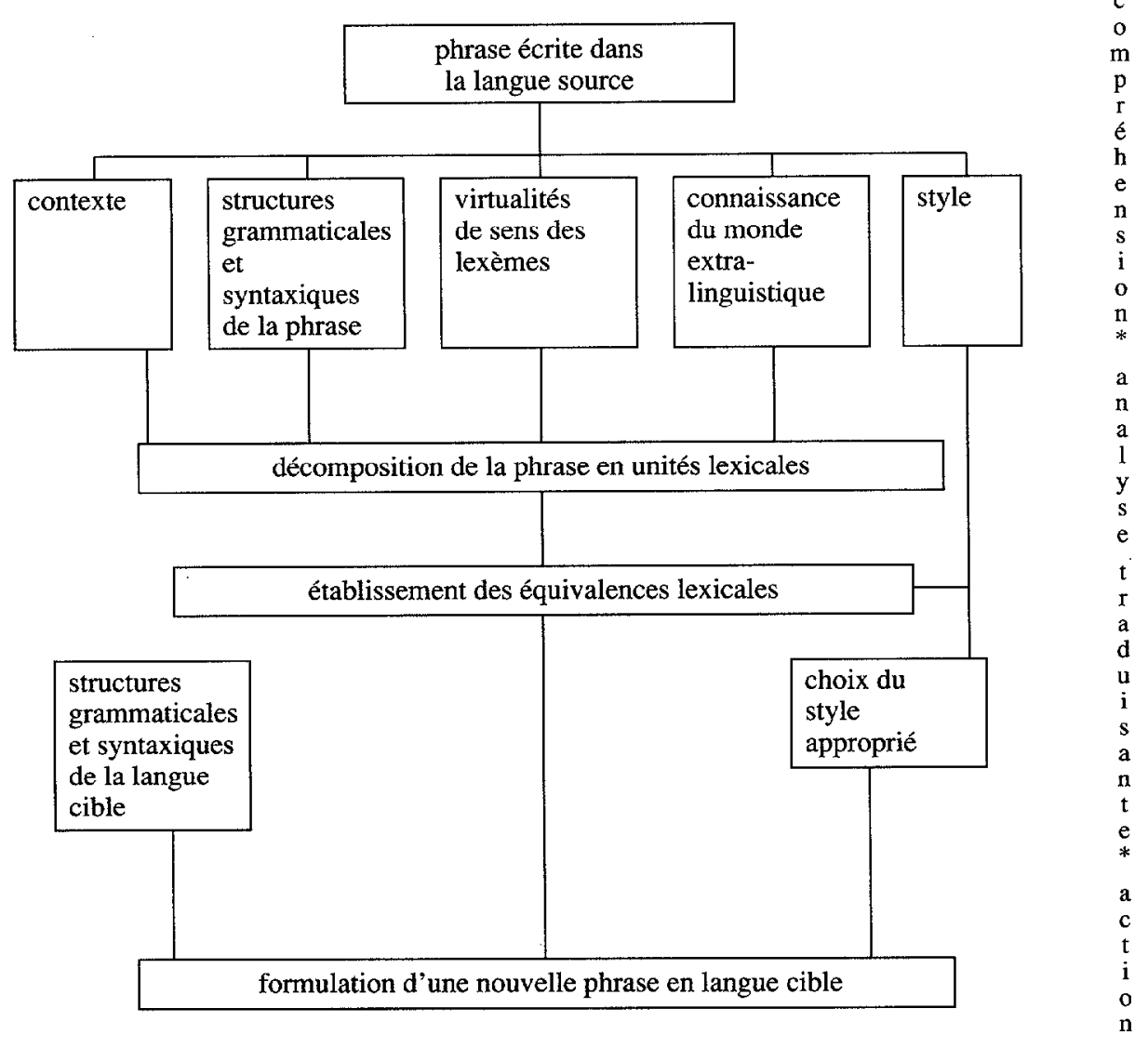




\section{BIBLIOGRAPHIE}

ALSTON, W. P. (1971) : «How Does One Tell Whether a Word Has One, Several or Many Senses ?», Steinberg, D.D. et L.A. Jakobovits (eds.), Semantics, Cambridge, Cambridge University Press, pp. 35-47.

ANDERSON, J. D. (1972): «The Development of the English-French, French-English Bilingual Dictionary: A Study in Comparative Lexicography», Word, 8, pp. 1-144.

COHEN, M. (1962) : «Le fait dictionnaire», Proceedings of the 9th International Congress of Linguists, The Hague, pp. 497-503.

CRUSE, D. A. (1986): Lexical Semantics, Cambridge, Cambridge University Press.

DELISLE, J. (1984): L'Analyse du discours comme méthode de traduction, Ottawa, Éditions de l'Université d'Ottawa.

HARTMANN, R. R. K. (1983) : «Introductory Essay», Proceedings of "Lexeter $83 »$, Exeter, Niemeyer.

HOUSEHOLDER, F. W. and S. SAPORTA (eds.) (1975): Problems in Lexicography, Bloomington, Indiana University Publications.

KATZ, J. J. et J. A. FODOR (1963) : «The Structure of a Semantic Theory», Language, 39, pp. 170-210.

LAROSE, R. (1987): Théories contemporaines de la traduction, Sillery, Presses de l'Université du Québec.

LEDERER, M. (1973) : «La traduction: transcoder ou réexprimer ?», Études de linguistique appliquée, 12, Paris, Didier.

MOSKOWITZ, D. (1973) : «Le traducteur : récepteur et destinataire du message», Études de linguistique appliquée, 12, Paris, Didier.

SAUSSURE, F. de (1973) : Cours de linguistique générale, édition critique préparée par Tullio de Mauro, Paris, Payot.

STEINBERG, D. D. and L.A. JAKOBOVITS (eds.) (1971): Semantics, Cambridge, Cambridge University Press.

TATILON, C. (1982) : «Traitement des unités lexicales», Meta 27-2.

VINAY, J.-P. et J. DARBELNET (1977): Stylistique comparée du français et de l'anglais, Montréal, Beauchemin.

WEINREICH, U. (1975) : «Lexicographic Definition in Descriptive Semantics», Householder, F. W. and S. Saporta (eds.), Problems in Lexicography, pp. 25-44.

WIEZELL, R. J. (1975) : «Problems in bilingual lexicography: Romance and English», Hispania, 58-1, pp. 131136.

WIGGINS, D. (1971): «On Sentence-sense, Word-sense and Difference of Word-sense. Towards a Philosophical Theory of Dictionaries», Semantics, Cambridge, Cambridge University Press, pp. 14-34. 